Conclusions This study demonstrates a latent class modelling approach for IA diagnosis in LTR with a combination of culture, composite biomarker testing, and radiology required for optimal IA diagnosis.

\section{S18 MECHANISMS REGULATING COLLAGENOLYTIC AND ELASTOLYTIC ACTIVITY IN M. AVIUM INFECTION}

SJ McFetridge, R McMullan, GN Schroeder, CM O'Kane. Queen's University Belfast, Belfast, UK

\subsection{6/thoraxjn--2017-210983.24}

Background The incidence of pulmonary non-tuberculous mycobacterial (NTM) infection is increasing. In the UK mycobacterium avium complex (MAC), is the commonest NTM infection outside of CF lung disease. Patients with pulmonary MAC infection develop cavitating lung disease or nodular bronchiectasis, but the mechanisms of tissue destruction are not well-characterised, unlike $M$. tuberculosis infection. We have previously shown that clinical isolates of M. avium surprisingly do not drive secretion of MMP-9 by infected macrophages. Instead, M. avium drives functionally unopposed MMP-1, previously thought to be an M. tuberculosis-specific response, and MMP-7. We investigated the mechanisms regulating MAC-induced MMP-1 and -7 secretion.

Methods Monocytes were isolated from healthy human volunteer blood by density centrifugation and adherence, before incubation in GM-CSF for 7 days to generate monocytederived macrophages (MDMs). MDMs were stimulated with four different clinical isolates of M. avium at MOI 100 for up to 72 hours. Whole cell lysates, and cytoplasmic and nuclear extracts were collected 15 mins -4 hours after infection, and analysed by western blot for protein phosphorylation or TransAm assay for NF- $\kappa \mathrm{B}$ activation. Supernatants collected at 72 hours were analysed by ELISA for MMP-1 and 7 .

Results Infection with $M$. avium caused activation of all 3 MAPK (p38, JNK, ERK) pathways as early as $15 \mathrm{~min}$ post exposure with maximal phosphorylation at $30 \mathrm{~min}$. M. avium infection drove maximal nuclear translocation of $\mathrm{NF}-\kappa \mathrm{B}$ and degradation of cytosolic $\mathrm{I} \kappa \mathrm{B} \alpha$ at $30 \mathrm{~min}$, returning to baseline by 4 hours. M. avium-induced MMP-1 secretion from MDMs is ERK and JNK, but not p38- dependant (figure 1). Treat-

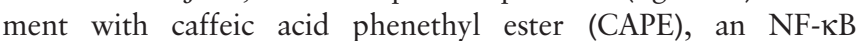
inhibitor, reduced $M$. avium-induced MMP-1 secretion by 30\%. Both MMP-1 and -7 upregulation were suppressed by PI3 kinase inhibitor LY294002. M. avium-induced MMP-7 upregulation was not inhibited by indomethacin.

Conclusions MMP-1 and -7 may drive the destructive pulmonary pathophysiology that characterises MAC infection. However, regulation of the host macrophage response to M. avium is divergent to that M. tuberculosis, with p38- independent MMP-1 secretion. This divergence in intracellular signalling may necessitate deviation in potential adjunctive patient therapies for M. tuberculosis and M. avium.

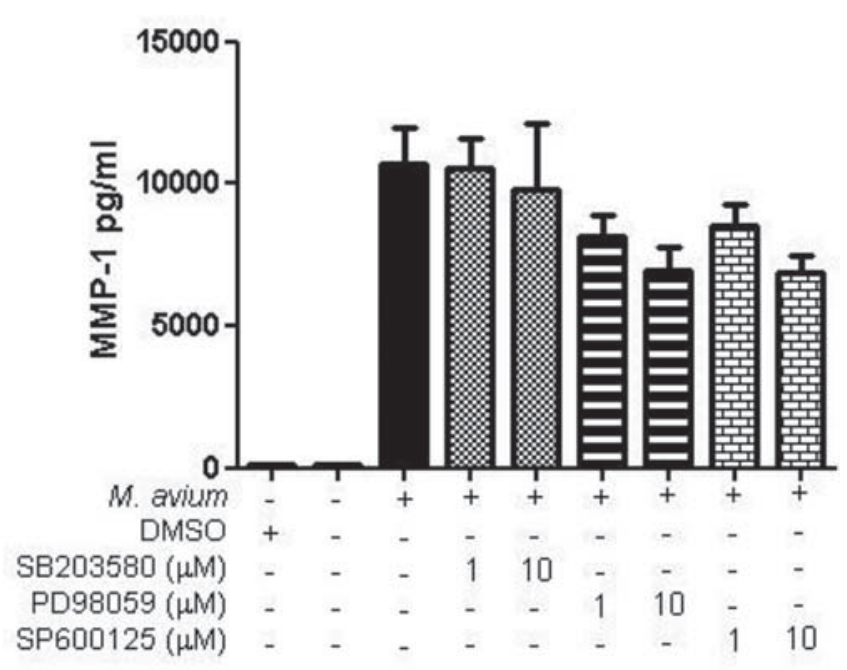
stimulated MDMs, pre-incubated with p38, ERK or JNK inhibitor prior to infection. Supernatants were harvested at 72 hours and MMP concentration analysed by ELISA.

\section{S19 THE ROLE OF LYMPH NODE-RESIDENT NEUTROPHILS IN ADAPTIVE IMMUNITY}

LSC Lok, B Stewart, ER Chilvers, MR Clatworthy. Department of Medicine, University of Cambridge, Cambridge, UK

\subsection{6/thoraxjnl-2017-210983.25}

Introduction Neutrophils play a key role in the early response to a diverse range of infectious and inflammatory stimuli. However, persistent neutrophilic inflammation can result in collateral tissue damage, as evident in a number of chronic respiratory diseases. In addition to their role in innate immunity, neutrophils can also shape the adaptive immune response, in part through antigen presentation. Whilst there is accumulating evidence that neutrophils can migrate to draining lymph 